\title{
The Content Types Dataset: a New Resource to Explore Semantic and Functional Characteristics of Texts
}

\author{
Rachele Sprugnoli ${ }^{1-3}$, Tommaso Caselli $^{2}$, Sara Tonelli ${ }^{1}$ and \\ Giovanni Moretti ${ }^{1}$ \\ ${ }^{1}$ DH-FBK, Via Sommarive 18, Povo, Trento \\ ${ }^{2}$ CLTL, Vrije Univeristieit Amsterdam, De Boelelaan, 1105, Amsterdam \\ ${ }^{3}$ University of Trento, Via Sommarive 9, Povo, Trento \\ \{sprugnoli, satonelli, moretti\}@fbk.eu \\ $\{$ t.caselli\}evu.nl
}

\begin{abstract}
This paper presents a new resource, called Content Types Dataset, to promote the analysis of texts as a composition of units with specific semantic and functional roles. By developing this dataset, we also introduce a new NLP task for the automatic classification of Content Types. The annotation scheme and the dataset are described together with two sets of classification experiments.
\end{abstract}

\section{Introduction}

This paper introduces a new resource and task for NLP, namely the classification of Content Types. The notion of Content Types differs from standard discourse relations, either based on rhetorical structures or lexically-grounded approaches. Content Types provide cues to access the structure of a document's types of functional content. They contribute to the overall message or purpose of a text and make explicit the functional role of a discourse segment with respect to its content, i.e. meaning. Their identification may improve the performance of more complex NLP tasks by targeting the portions of the documents that are more relevant. For example, when building a storyline it may be useful to focus on the narrative segments of a text (Vossen et al., 2015), while for sentiment analysis the identification of evaluative clauses may be beneficial (Liu, 2015).

Our contribution is threefold: i) we make available annotation guidelines with high reliability in terms of inter-annotator agreement and applicable to texts of different genres and period of publication; ii) we release the first version of a new dataset (whose annotation is still in progress) that takes into consideration both contemporary and historical texts, paving the way to a new NLP task, i.e.
Content Type Classification; and iii) we present initial promising results for the automatic classification of Content Types by using the first version of the dataset. All data are made available on-line ${ }^{1}$.

The remainder of the paper is structured as follows: Section 2 illustrates the annotation scheme, the composition of the dataset, and report the interannotator agreement. Section 3 presents two sets of experiments to automatically classify Content Types. Related work is discussed in Section 4. Finally, conclusion and future work are reported in Section 5.

\section{Dataset Construction}

Content Types (henceforth CTs) are text passages with specific semantic and functional characteristics. Their definition is based on linguistic features, and the annotation is performed at clause level. Clauses are considered as textual constituent units (Polanyi, 1988), and defined as groups of words related to each other, containing a finite or non-finite verb, while the subject may be implicit or shared with other clauses. This granularity level of the mark-up was chosen to provide a fine-grained annotation of CTs that can characterize different portions of the same sentence. Example (1) is made of two clauses (divided by "//"): the first narrates what the author is doing, the second describes the place where she is.

(1) I am writing on a fine terrace overlooking the sea,// where stone benches and tables are conveniently arranged for our use.

We identify seven classes of CTs, five of which are based from Werlich's typology, while the last two (OTHER and NONE) were introduced in our

\footnotetext{
${ }^{1}$ https://github.com/dhfbk/ content-types
} 


\begin{tabular}{lcc}
\hline & News & $\begin{array}{c}\text { Travel } \\
\text { Reports }\end{array}$ \\
\hline Evaluative & 0.82 & 0.90 \\
Descriptive & 0.84 & 0.86 \\
Expository & - & 0.93 \\
Instructive & - & 0.65 \\
Narrative & 0.86 & 0.88 \\
None & 1.0 & 1.0 \\
Other & - & 0.92 \\
\hline
\end{tabular}

Table 1: Inter Annotator Agreement: Cohen's kappa calculated at token level.

scheme to account for undefined or unclear cases:

- NARRATIVE: clauses containing events and states that can be anchored to a hypothetical timeline; e.g., We left Cava on Wednesday,// and made the tour from there to Amalfi.

- EVALUATIVE: clauses with explicit evaluation markers; e.g., Telerate's two independent directors have rejected as inadequate.

- DESCRIPTIVE: clauses presenting tangible and intangible characteristics of entities, such as objects, persons or locations; e.g., The road winds above, beneath, and beside rugged cliffs of great height.

- EXPOSITORY: clauses expressing generalizations with respect to a class.; e.g., All Italians are dandies.

- INSTRUCTIVE: clauses expressing procedural information; e.g., At last you cross that big road // and strike the limestone rock.

- OTHER: clauses containing text in foreign languages, phatic expressions, references to the reader; e.g., Madame est servie.

- NONE: clauses that cannot be labeled with any of the previous classes; e.g., Chapter IV.

This specific set of classes was selected because it provides a good level of generalization for characterizing the contents of non-standardized documents (e.g. news articles $v s$. scientific article), and it can be applied across different domains and genres. Each markable has a set of attributes used to: (i) specify whether a CT is part of a direct or reported speech , (ii) distinguish digressions from the primary narration, (iii) indicate whether a description refers to a person, a location or another kind of entity, and (iv) typify the clauses annotated as OTHER.
To test the comprehensiveness of this scheme, we annotate English texts from two different genres and periods of publication: namely, contemporary news and travel reports published between the end of the XIX Century and the beginning of the XX Century. While the former are taken from already available datasets, i.e., TempEval-3, Penn Discourse Treebank, and MASC (UzZaman et al., 2013; Prasad et al., 2008; Ide et al., 2010), the latter constitute a novel set of texts extracted from the Gutenberg project ${ }^{2}$. The corpus is released under the name of Content Types Dataset version 1.0 (CTD_v1). The resource is still being extended with new annotated texts, but in the remainder of the paper we will refer to this first version.

The annotation was conducted by two expert linguists following a multi-step process and using the web-based tool CAT (Bartalesi Lenzi et al., 2012). In the first phase, annotators were allowed to discuss disagreements based on a trial corpus suggesting revisions to improve the guidelines. In the second phase, inter-annotator agreement was calculated on a subset of the CTD_v1 (a total of 5,328 tokens and 526 clauses, with 2,500 tokens and about 250 clauses per genre). Table 1 reports the Cohen's kappa on the number of tokens for both text genres. With the exception of the INSTRUCTIVE CT, all the classes have high scores, exceeding 0.8 , usually set as a threshold that guarantees good annotation quality (Artstein and Poesio, 2008). In the final phase, the whole dataset was annotated using the latest version of the guidelines which includes detailed descriptions of the classes, examples for both genres, and priority rules discriminating when more than one CT class may apply to clauses. Table 2 illustrates the composition of CTD_v1. The two genres of texts show, for almost all the CT classes, a statistically significant difference (at $p<0.01$ and calculated with the $\mathrm{z}$ test) in their distribution.

\section{Experiments}

In this section we present initial experiments for the automatic classification of clauses in CTs. Attribute classification was not targeted at this stage. We conducted two sets of experiments to test different modeling assumptions. In all experiments we use gold clause boundaries.

\footnotetext{
${ }^{2}$ http://www - gutenberg . org/
} 


\begin{tabular}{l|llll}
\hline & & News & Travel Reports & Total \\
\hline & Texts & 84 & 25 & 109 \\
& Tokens & 32,086 & 31,715 & 63,801 \\
& Clauses & 3,038 & 3,158 & 6,196 \\
\hline \multirow{3}{*}{} & Evaluative* & $428(14.09 \%)$ & $618(19.59 \%)$ & $1,046(16.88 \%)$ \\
& Descriptive* & $198(6.52 \%)$ & $480(15.19 \%)$ & $678(10.94 \%)$ \\
& Expository & $58(1.91 \%)$ & $81(2.56 \%)$ & $139(2.24 \%)$ \\
\multirow{\Xi}{\Xi}{} & Instructive & $5(0.16 \%)$ & $4(0.13 \%)$ & $9(0.15 \%)$ \\
& Narrative* & $2,318(76.30 \%)$ & $1,738(55.03 \%)$ & $4,056(65.46 \%)$ \\
& None* & $15(0.49 \%)$ & $38(1.20 \%)$ & $53(0.86 \%)$ \\
& Other* & $16(0.53 \%)$ & $199(6.30 \%)$ & $215(3.47 \%)$ \\
\hline
\end{tabular}

Table 2: Statistics of $C T D_{-} v 1$ : an asterisk indicates whether the content type has a statistically significant difference in the distribution over the two genres.

\begin{tabular}{l|l}
\hline Clause Component & Features \\
\hline Noun Phrase & $\begin{array}{l}\text { phrase tokens, head token, head lemma, determiner type, person, number, } \\
\text { countability,head type, head POS, WordNet sense and supersense, WordNet } \\
\text { hypernyms, length of path to the top node in WordNet }\end{array}$ \\
\hline Verb Phrase & $\begin{array}{l}\text { phrase tokens, head token, head lemma, clause adverb, lemma of clause adverb, } \\
\text { coarse tense values (present, past, future), fine-grained tense values (present } \\
\text { perfect, etc.), voice, grammatical aspect (progressive, perfect), WordNet sense } \\
\text { and supersense, WordNet hypernyms, length of path to the top node in } \\
\text { WordNet, head POS }\end{array}$ \\
\hline
\end{tabular}

Table 3: Features of the clause components.

\subsection{Feature Sets}

We experiment two different types of features: the first relies on distributional information extracted through sentence embeddings (Le and Mikolov, 2014), while the second is linguistically motivated and focuses on syntactic and semantic properties of the main components of the clause, i.e. the noun phrase(s) and the verb phrase. For the first type, we extracted embeddings for each clause using the doc2vec (Le and Mikolov, 2014) implementation in gensim, with vector size $=50$ and window $=5$. For the second feature type, all documents were pre-processed at clause level with Stanford CoreNLP (Manning et al., 2014), performing tokenization, lemmatization, POS tagging, Named Entity recognition. The extraction of basic syntactic and semantic properties of the clause components has been performed with a syntactic-semantic features toolkit (Friedrich and Pinkal, 2015). This has allowed us to identify four blocks of features for: (i) the noun phrase in subject position (i.e. nsubj and nsubjpass), (ii) the noun phrase in direct object position (i.e. dob $j$ and agent), (iii) the noun phrase in any other syntactic relation, and (iv) the clause verb. Details for noun phrase and verb phrase components are reported in Table 3.

We extended the basic features with prior sentiment polarity scores for nouns, verbs, adjectives, and adverbs in the clause via SentiWordNet (Baccianella et al., 2010). For each target POS, polarity scores are aggregated per lemma and averaged by the number of senses, thus providing a lemma-based prior polarity. Finally, the lemma-based polarity scores are normalized by the clause length and scaled between 0 and 1 . Finally, we introduced a binary feature to mark the presence/absence of a temporal expression in a clause. These two additional blocks of features have been selected following the definition of the CTs in the annotation guidelines. In particular, the presence of temporal expressions in a clause can facilitate the distinction between the NARRATIVE and the DESCRIPTIVE classes, while the polarity features should facilitate the identification of the EVALUATIVE class. 


\subsection{Classification Experiments}

We developed our models by dividing the annotated data in training (80\%) and test sections (20\%), balancing the distribution in each section across the two genres. The overall amount of clauses in the training and test data is slightly lower than the one of the manually annotated clauses $^{3}$ : indeed, we excluded some clauses because the pre-processing tools were not able to extract any relevant features from them. This is mainly due to a failure of the syntactic-semantic toolkit to process some gold clauses.

To better evaluate the performance of our models, we developed a baseline system by assigning the most frequent $\mathrm{CT}$ per text genre on the basis of the frequencies in the training data. Evaluation has been computed by means of Precision, Recall, and F1-score as implemented in scikit-learn (Pedregosa et al., 2011).

Content-based Classification In this set of experiments we aimed at verifying the fitness of our features by assuming that CTs are independent of each other and determined only by their meaning. We developed four classifiers, by varying the combination of features, using two different learners, namely Support Vector Machines (SVM) (Cortes and Vapnik, 1995) and Conditional Random Fields (CRFs) (Lafferty et al., 2001):

- clause model has only basic clause features plus the polarity scores and the presence/absence of temporal expressions.

- clause+doc2vec model has the clause model feature set extended with the doc2vec clause embeddings.

The SVM models have been implemented using LIBSVM (Chang and Lin, 2011) with Linear Kernel. The CRF models have been implemented with $\mathrm{CRF}++$ toolkit ${ }^{4}$ with default parameters.

Content and Functional Structure Classification This set of experiments assumes an alternative modeling strategy by viewing each sentence as a sequence of CTs, each associated with a clause. For this second set of experiments we implemented two linear CRF classifiers by extending the previously described models with a context window of [+/-2] for all features.

\footnotetext{
${ }^{3} 5,503$ vs. 5,536 in the training set; 653 vs. 660 in the test set.

${ }^{4}$ https://taku910.github.io/crfpp/
}

Results are illustrated in Table 4. The content-based classification experiments show that CTs are subject to the functional structure of the sentence and, more generally, of the document. Only the CRF classifiers, i.e. sequence labeling models, can beat the baseline, providing balanced results for Precision and Recall, and improving the F1 score by 0.11 (CRF-clauseC) and 0.10 points (CRF-clause+doc2vecC). The SVM models, on the contrary, fail to beat the baseline. This could be due to the imbalanced distribution of CTs, and also to the fact that content features alone are not enough to discriminate the different CTs. The contribution of the doc2vec features is, however, limited: they help increasing the Recall values (+0.03 points) but have a little effect on the Precision (+0.01 point) when considering the CRF models. On the contrary, they do not provide any improvements with the SVM models.

As for the content and functional structure classification models, the results indicate that context features positively contribute to the improvement of the classification task (the CRFClauseCF with context features outperforms its direct counterpart, CRF-clauseC, in the content-based classification setting). It is interesting to notice a redundancy between the doc $2 \mathrm{vec}$ features and the context window. In this case, the CRF-clause+doc2vecCF has the lowest results for Precision and F1, and a slight increase in Recall (0.68 vs. 0.67).

\section{Related Work}

The classification of text passages has been studied in previous works considering different textual units (e.g., clauses, sentences, and paragraphs) or language patterns (Kaufer et al., 2004). Several annotation schemes, often based on genre-specific taxonomies, have been proposed. This is the case, for example, of the detection of the main components in scholarly publications (Teufel et al., 2009; Liakata et al., 2012; De Waard and Maat, 2012; Burns et al., 2016) or the annotation of content zones, i.e., functional constituents of texts (Bieler et al., 2007; Stede and Kuhn, 2009; Baiamonte et al., 2016). On the contrary, the notion of Content Types that we have adopted applies across genres. CTs are based on linguistic theories on discourse/rhetorical strategies but differ from discourse relations. Over the years, different typologies have been proposed (Werlich, 1976; Biber, 


\begin{tabular}{l|llll}
\hline \multicolumn{5}{c}{ Content-based Classification } \\
\hline Model & $\mathrm{P}$ & $\mathrm{R}$ & $\mathrm{F} 1$ & Acc. \\
\hline Baseline (NARRATIVE) & 0.42 & 0.65 & 0.51 & 0.65 \\
SVM-clause & 0.42 & 0.65 & 0.51 & 0.65 \\
SVM-clause+doc2vec & 0.42 & 0.65 & 0.51 & 0.65 \\
CRF-clauseC & 0.61 & 0.65 & 0.62 & 0.66 \\
CRF-clause+doc2vecC & 0.62 & 0.68 & 0.61 & 0.67 \\
\hline \multicolumn{4}{c}{ Content and Functional Structure Classification } \\
\hline Model & $\mathrm{P}$ & $\mathrm{R}$ & $\mathrm{F} 1$ & Acc. \\
\hline Baseline (NARRATIVE) & 0.42 & 0.65 & 0.51 & 0.65 \\
CRF-clauseCF & 0.62 & 0.67 & 0.64 & 0.67 \\
CRF-clause+doc2vecCF & 0.60 & 0.68 & 0.61 & 0.68 \\
\hline
\end{tabular}

Table 4: Results of the classification experiments.

1989; Chatman, 1990; Adam, 1985; Longacre, 2013) but have been rarely treated computationally, with the exception of the work by Cocco et al. (2011).

The theory of Discourse Modes (DMs) (Smith, 2003) is instead followed by Mavridou et al. (2015) that apply it to a paragraph-based pilot annotation of a variety of documents such as novels, news and European Parliament proceedings. Annotators intuitively labeled DMs relying on a very short manual: as a consequence, no formal guidelines were made available and only a moderate agreement was achieved. Moreover, the final dataset is not publicly available and the recognition of DMs has not been automated yet. Our approach is different: we rely on Werlich's typology, we provide complete annotation guidelines, we make available the annotated dataset, and we experiment automatic classification of CTs.

\section{Conclusion and Future Work}

In this work, we presented a novel resource annotated with CTs and a set of experiments aimed at automatically classifying clauses based on content and on their functional structure. Although this work is still in progress, the proposed annotation scheme proved sound and the developed corpus can already provide insights into the functional role of discourse segments with respect to the clause meaning.

In addition to SVM and CRFs, we experimented with artificial neural networks (ANN) using the Keras ${ }^{5}$ framework running on the TensorFlow implementation (Abadi et al., 2015). We tested different configurations but results are not higher

\footnotetext{
${ }^{5}$ https://github.com/fchollet/keras
}

than those obtained with CRFs. We will investigate the reasons and try other models. Similarly, we will investigate whether SVM kernels other than the linear one can do better.

In the future, we will continue the annotation of the dataset, by introducing documents from other text genres (e.g. travel guides, news editorials, school textbooks) so as to re-balance the distributions of the CTs in the dataset. Furthermore, we plan to study whether information on content types can contribute to other NLP tasks. For example, we believe that identifying NARRATIVE and EVALUATIVE CTs may contribute to discriminating between clauses useful to build a storyline or a timeline of events (the former) and clauses bearing sentiment information (the latter).

\section{Acknowledgement}

Part of this work has been conducted during a visiting period of one of the author at the Computational Lexicology \& Terminology Lab (CLTL) at the Vrije Universiteit Amsterdam. One of the author wants to thanks the NWO Spinoza Prize project Understanding Language by Machines (sub-track 3 ) for supporting this work.

\section{References}

Martın Abadi, Ashish Agarwal, Paul Barham, Eugene Brevdo, Zhifeng Chen, Craig Citro, Greg S. Corrado, Andy Davis, Jeffrey Dean, Matthieu Devin, et al. 2015. TensorFlow: Large-scale machine learning on heterogeneous systems. Software available from tensorflow. org, 1.

Jean-Michel Adam. 1985. Quels types de textes?(What Kinds of Text?). Français dans le monde, 192:39-43. 
Ron Artstein and Massimo Poesio. 2008. Inter-coder agreement for computational linguistics. Computational Linguistics, 34(4):555-596.

Stefano Baccianella, Andrea Esuli, and Fabrizio Sebastiani. 2010. SentiWordNet 3.0: An Enhanced Lexical Resource for Sentiment Analysis and Opinion Mining. In LREC, volume 10, pages 2200-2204.

Daniela Baiamonte, Tommaso Caselli, and Irina Prodanof. 2016. Annotating Content Zones in News Articles. In Proceedings of Third Italian Conference on Computational Linguistics (CLiC-it 2016) \& Fifth Evaluation Campaign of Natural Language Processing and Speech Tools for Italian. Final Workshop (EVALITA 2016). Associazione Italiana di Linguistica Computazionale.

Valentina Bartalesi Lenzi, Giovanni Moretti, and Rachele Sprugnoli. 2012. CAT: the CELCT Annotation Tool. In In Proceedings of LREC 2012, pages 333-338.

Douglas Biber. 1989. A typology of english texts. Linguistics, 27(1):3-44.

Heike Bieler, Stefanie Dipper, and Manfred Stede. 2007. Identifying formal and functional zones in film reviews. Proceedings of the 8th SIGDIAL, pages $75-78$.

Gully APC Burns, Pradeep Dasigi, Anita de Waard, and Eduard H. Hovy. 2016. Automated detection of discourse segment and experimental types from the text of cancer pathway results sections. Database, 2016:baw122.

Chih-Chung Chang and Chih-Jen Lin. 2011. LIBSVM: a library for support vector machines. $A C M$ Transactions on Intelligent Systems and Technology (TIST), 2(3):27.

Seymour Benjamin Chatman. 1990. Coming to terms: the rhetoric of narrative in fiction and film. Cornell University Press.

Christelle Cocco, Raphaël Pittier, François Bavaud, and Aris Xanthos. 2011. Segmentation and Clustering of Textual Sequences: a Typological Approach. In $R A N L P$, pages $427-433$.

Corinna Cortes and Vladimir Vapnik. 1995. Supportvector networks. Machine learning, 20(3):273-297.

Anita De Waard and Henk Pander Maat. 2012. Epistemic modality and knowledge attribution in scientific discourse: A taxonomy of types and overview of features. In Proceedings of the Workshop on Detecting Structure in Scholarly Discourse, pages 4755. Association for Computational Linguistics.

Annemarie Friedrich and Manfred Pinkal. 2015. Discourse-sensitive Automatic Identification of Generic Expressions. In Proceedings of the 53rd Annual Meeting of the Association for Computational Linguistics (ACL).
Nancy Ide, Christiane Fellbaum, Collin Baker, and Rebecca Passonneau. 2010. The manually annotated sub-corpus: A community resource for and by the people. In Proceedings of the ACL 2010 conference short papers, pages 68-73. Association for Computational Linguistics.

David S. Kaufer, Suguru Ishizaki, Brian S. Butler, and Jeff Collins. 2004. The Power of Words: Unveiling the Speaker and Writer's Hidden Craft. Routledge.

John Lafferty, Andrew McCallum, and Fernando Pereira. 2001. Conditional random fields: Probabilistic models for segmenting and labeling sequence data. In Proceedings of the eighteenth international conference on machine learning, ICML, volume 1, pages 282-289.

Quoc V. Le and Tomas Mikolov. 2014. Distributed Representations of Sentences and Documents. In ICML, volume 14 , pages $1188-1196$.

Maria Liakata, Shyamasree Saha, Simon Dobnik, Colin Batchelor, and Dietrich Rebholz-Schuhmann. 2012. Automatic recognition of conceptualization zones in scientific articles and two life science applications. Bioinformatics, 28(7):991-1000.

Bing Liu. 2015. Sentiment analysis: Mining opinions, sentiments, and emotions. Cambridge University Press.

Robert E. Longacre. 2013. The grammar of discourse. Springer Science \& Business Media.

Christopher D. Manning, Mihai Surdeanu, John Bauer, Jenny Finkel, Steven J. Bethard, and David McClosky. 2014. The Stanford CoreNLP natural language processing toolkit. In Association for Computational Linguistics (ACL) System Demonstrations, pages 55-60.

Kleio-Isidora Mavridou, Annemarie Friedrich, Melissa Peate Sørensen, Alexis Palmer, and Manfred Pinkal. 2015. Linking discourse modes and situation entity types in a cross-linguistic corpus study. In Workshop on Linking Models of Lexical, Sentential and Discourse-level Semantics (LSDSem), page 12.

Fabian Pedregosa, Gaël Varoquaux, Alexandre Gramfort, Vincent Michel, Bertrand Thirion, Olivier Grisel, Mathieu Blondel, Peter Prettenhofer, Ron Weiss, Vincent Dubourg, et al. 2011. Scikit-learn: Machine learning in python. Journal of Machine Learning Research, 12(Oct):2825-2830.

Livia Polanyi. 1988. A formal model of the structure of discourse. Journal of pragmatics, 12(5-6):601638.

Rashmi Prasad, Nikhil Dinesh, Alan Lee, Eleni Miltsakaki, Livio Robaldo, Aravind K. Joshi, and Bonnie L. Webber. 2008. The Penn Discourse TreeBank 2.0. In $L R E C$. Citeseer. 
Carlota S. Smith. 2003. Modes of discourse: the local structure of texts, volume 103. Cambridge University Press.

Manfred Stede and Florian Kuhn. 2009. Identifying the content zones of German court decisions. In International Conference on Business Information Systems, pages 310-315. Springer.

Simone Teufel, Advaith Siddharthan, and Colin Batchelor. 2009. Towards discipline-independent argumentative zoning: evidence from chemistry and computational linguistics. In Proceedings of the 2009 Conference on Empirical Methods in Natural Language Processing: Volume 3-Volume 3, pages 1493-1502. Association for Computational Linguistics.

Naushad UzZaman, Hector Llorens, Leon Derczynski, James Allen, Marc Verhagen, and James Pustejovsky. 2013. Semeval-2013 task 1: Tempeval-3: Evaluating time expressions, events, and temporal relations. In Second Joint Conference on Lexical and Computational Semantics (*SEM), Volume 2: Proceedings of the Seventh International Workshop on Semantic Evaluation (SemEval 2013), pages 1-9, Atlanta, Georgia, USA, June. Association for Computational Linguistics.

Piek Vossen, Tommaso Caselli, and Yiota Kontzopoulou. 2015. Storylines for structuring massive streams of news. In Proceedings of the First Workshop on Computing News Storylines, pages 40-49.

Egon Werlich. 1976. A text grammar of English. Quelle \& Meyer. 